\title{
Optimizing Mesostructured Silver Catalysts for Selective Carbon Dioxide Conversion into Fuels
}

\author{
Silvan Suter ${ }^{a}$, Sophia Haussener ${ }^{a, 1}$ \\ aLaboratory of Renewable Energy Science and Engineering, EPFL, Station 9, 1015 Lausanne, Switzerland
}

\section{Abstract}

Mesostructured silver catalysts have shown a substantial rise in reaction selectivity for the conversion of $\mathrm{CO}_{2}$ into fuels with increasing thickness of ordered, inverse-opal films. The challenge lies in the optimization of the morphology to maximize the $\mathrm{CO}$ selectivity. We developed a 3D mass transport model utilizing the accurate electrode geometry to calculate local concentration distributions of $\mathrm{CO}_{2}(\mathrm{aq}), \mathrm{OH}^{-}, \mathrm{HCO}_{3}{ }^{-}$, and $\mathrm{CO}_{3}{ }^{2-}$ by considering the buffer reactions in the electrolyte and modeling local catalytic surface reaction rates based on Butler-Volmer correlations. Validated with experimental data from the literature, the model predicted the suppression of the hydrogen evolution reaction with an inverse dependency on the hydroxide concentration and the promotion of the $\mathrm{CO}$ evolution reaction with a proportional dependency on the carbonate concentration. In order to increase the CO selectivity, we developed design guidelines that suggest high electrode roughness per film thickness, which translates to smaller pore size in practice. Further, the shallow pores of the electrode strongly reduced the overall CO selectivity as the mass transport to the bulk was non-limiting. We demonstrated that the introduction of an additional diffusion layer on top of the silver electrode can enhance the CO selectivity from as low as $39 \%$ to more than $90 \%$.

\section{Introduction}

The electrochemical reduction of $\mathrm{CO}_{2}$ offers a path to store electricity from renewable sources in energy-dense chemical bonds. ${ }^{1-4}$ During this process, carbon will be recycled by transforming available $\mathrm{CO}_{2}$ into valuable chemicals and fuels while mitigating further greenhouse gas emissions. ${ }^{5-7}$ Transition metals (e.g., $\mathrm{Cu}, \mathrm{Au}, \mathrm{Ag}$ ) have been demonstrated to catalyze the multi-electron, electrochemical reduction of $\mathrm{CO}_{2}$ for the production of carbon monoxide (CO), formic acid or formate $(\mathrm{HCOOH}$ or $\mathrm{HCOO}^{-}$), hydrocarbons and alcohols. ${ }^{6,8}$ However, low energy efficiencies and high costs have so far been the obstacles for novel $\mathrm{CO}_{2}$-to-fuel conversion technologies. ${ }^{3}$ In addition, the selectivity of the catalysts towards the desired products is often poor, which adds to the complexity of the system. ${ }^{9}$ In most practical applications, the $\mathrm{CO}_{2}$ is reduced in an aqueous environment, where the hydrogen evolution reaction rate often outcompetes the desired product evolution rate. ${ }^{4}$ Unless the goal is to produce synthesis gas (a mixture of hydrogen and $\mathrm{CO})^{10}$, the key requirement for an efficient $\mathrm{CO}_{2}$-to-fuel catalyst is to favor the activation of $\mathrm{CO}_{2}$ to produce $\mathrm{CO}$ and keep the hydrogen evolution rate low. Even if $\mathrm{CO}$ is further reduced on a copper surface, the initial selectivity towards $\mathrm{CO}$ will determine the overall upper limit for the $\mathrm{CO}_{2}$-to-fuel conversion. ${ }^{11-13}$ Gold and silver electrodes, in particular, have shown to reduce $\mathrm{CO}_{2}$ only to $\mathrm{CO}$, but the selectivity for this reaction is low compared to the hydrogen evolution reaction. ${ }^{14-16}$ The selectivity depends on the intrinsic property of the active site of the catalyst surface and the local concentration of the reaction partners, which are the concentrations of reactants and species involved in the rate-limiting steps of the reaction pathway. ${ }^{17}$ The intrinsic properties are material specific, but research has demonstrated the impact of particle shape and size, and low-coordinated facets on the activity of the catalysts, allowing to tune the selectivity by nanostructuring. ${ }^{18}$ The species concentrations, on the other hand, can be influenced by the morphology on the mesoscale. An ordered mesostructure of gold inverse opals has proven to diminish the hydrogen evolution reaction by 10-fold compared to a planar electrode. ${ }^{19}$ By electrodepositing gold in the void of a close-packed colloidal polystyrene thin films, the surface structure remained unchanged for porous electrodes of various thickness. Therefore, suppression of the hydrogen evolution reaction had been attributed to the mass transport rather than the surface structure. Similar mesostructures made out of silver

\footnotetext{
${ }^{1}$ Corresponding author. E-mail address: sophia.haussener@epfl.ch, tel.: +41 216933878.
} 
have shown not only suppression of the hydrogen evolution reaction, but also promoted the $\mathrm{CO}$ evolution reaction, increasing the CO selectivity even further. ${ }^{17}$ With the two competing surface reactions and multiple buffer reactions in the electrolyte, a mesostructure introduces complex diffusional gradients that could increase the overall selectivity rather than just be limiting, as it would be the case for a single product evolution reaction. ${ }^{19,20}$ The species concentration and the $\mathrm{pH}$ value in the mesostructure can be substantially different from the bulk electrolyte. ${ }^{21}$ Due to the production of hydroxide ions $\left(\mathrm{OH}^{-}\right)$, the local $\mathrm{pH}$ near the catalyst surface is increased, which is thought to change the adsorption behavior of reaction intermediates that could pose a mass transport limit for the hydrogen evolution reaction. ${ }^{22-24}$ Also, the local $\mathrm{pH}$ affects the equilibria of the acid-base reaction in the buffer solution, shifting towards carbonates and bicarbonates, thereby reducing the local concentration of $\mathrm{CO}_{2} \cdot{ }^{20,25}$ For a better understanding of the processes that lead to an enhanced $\mathrm{CO}$ selectivity in mesostructures, it is important to know the species concentration distributions in the porous catalysts. Experimentally, only the bulk concentrations and $\mathrm{pH}$ can easily be measured, The local concentration values at the electrolyte-catalyst interface are not accessible. Computational mass transport models can provide additional information on the processes taking place on the pore level. So far, many of the numerical studies have focused on the device level, modeling the mass transport on a macro scale between the cathode and anode. ${ }^{10,26-28}$ Numerical models that focus on the $\mathrm{pH}$ and species distribution near the catalyst surface have been implemented in 1D for planar electrodes and show that higher buffer strength has a negative effect on the CO selectivity. ${ }^{20,29}$ Even though the bulk pH is higher than in a low buffer strength electrolyte, the local surface $\mathrm{pH}$ is reduced by the buffer reaction. In comparison with experimental data, a more sophisticated 1D model on a planar gold surface has been used to determine the role of the bicarbonate in the rate-limiting step of $\mathrm{CO}_{2}$ reduction catalysis. ${ }^{14}$ Furthermore, Raciti et al. ${ }^{30}$ recently applied a mass transport model to a nanostructured copper electrode. Their study predicted the species concentration and local $\mathrm{pH}$ values between the copper nanowires by using effective transport properties in a 1D approach.

Here we report a transport model based on the detailed 3D mesostructure, taking into account the diffusion of species in the pore space, the buffer reactions in the electrolyte and surface reaction rates dependent on the local concentration of the reaction partners. The model was validated with experimental data from Yoon et al. ${ }^{17}$ The results of the study provide new insights into the local species concentration distributions and the local current distribution of the $\mathrm{CO}$ and $\mathrm{H}_{2}$ evolution reactions. The model was used to establish new design guidelines for ordered, mesostructured inverse opals catalysts to increase the $\mathrm{CO}$ faradaic efficiency in electrochemical $\mathrm{CO}_{2}$ reduction.

\section{Model Description and Governing Equations}

We consider a mesoporous silver electrode for the electrochemical reduction of $\mathrm{CO}_{2}$ in potassium bicarbonate $\left(\mathrm{KHCO}_{3}\right)$ electrolyte. Our model domain replicates an ordered Ag inverse opal (Ag-IO) electrode structure fabricated by Yoon et al. ${ }^{17}$ They have used colloidal polystyrene thin films as a template for the silver deposition. The polystyrene spheres have been stacked in a hexagonal close-packed arrangement, leading to a face centered cubic (FCC) lattice of the pore network. The details of the fabrication process (sphere deposition, sintering, and silver electrodeposition) and experimental electrode characterization can be found in their publication. ${ }^{17}$ The thickness of the Ag-IO films can be precisely controlled, and the uniform porosity, surface structure, and tortuosity make these films suitable for the experimental validation of our model. The model domain of the porous Ag-IO network with pores of about $200 \mathrm{~nm}$ in diameter is shown in Figure 1. Twelve channels interconnect each pore, with channel diameters of around $70 \mathrm{~nm}$ (extracted from SEM images ${ }^{17}$ ). The desired morphology was achieved by overlapping the spheres of a hexagonal close-packed bed. The starting radius of the spheres was $r_{\text {sphere }}=r_{\text {pore }}-d r$, and the sphere radii were subsequently increased by $d r=7 \mathrm{~nm}$. The domain symmetries are along the hexagonal prism walls. The key morphology parameters of the Ag-IO films are the film thickness, $t$, the pore diameter $d_{\text {pore, }}$ and the surface roughness factor, $\mathrm{RF}$, defined as the electrochemical active

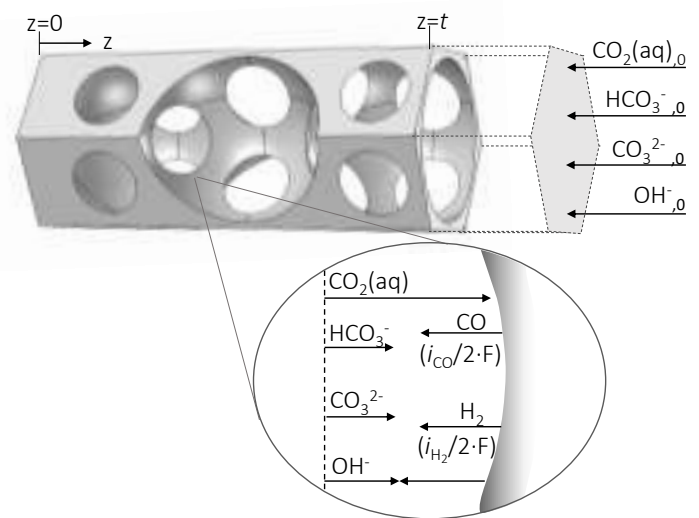

Figure 1. Illustration of the mass transport model for electrochemical reduction of $\mathrm{CO}_{2}$ on $\mathrm{Ag}-\mathrm{IO}$. 


\begin{tabular}{lll}
\hline \multicolumn{3}{l}{ Table 1. Rate constants for reactions (1) and (2) at $25^{\circ} \mathrm{C} .{ }^{20,31}$} \\
Reaction & Forward rate constant & Reverse rate constant \\
\hline 1 & $k_{1 \mathrm{f}}=5.93 \times 10^{3} \mathrm{M}^{-1} \mathrm{~s}^{-1}$ & $k_{1 \mathrm{r}}=1.34 \times 10^{-4} \mathrm{~s}^{-1}$ \\
2 & $k_{2 \mathrm{f}}=1 \times 10^{8} \mathrm{M}^{-1} \mathrm{~s}^{-1}$ & $k_{2 \mathrm{r}}=2.15 \times 10^{4} \mathrm{~s}^{-1}$ \\
\hline
\end{tabular}

Table 2. Species bulk concentrations in $0.1 \mathrm{M} \mathrm{KHCO}_{3}$ at $25^{\circ} \mathrm{C}^{14}$

\begin{tabular}{llll}
$\mathrm{CO}_{2}(\mathrm{aq})[\mathrm{M}]$ & $\mathrm{HCO}_{3}^{-}[\mathrm{M}]$ & $\mathrm{CO}_{3}{ }^{2-}[\mathrm{M}]$ & $\mathrm{OH}^{-}[\mathrm{M}]$ \\
\hline 0.033 & 0.1 & $2.9 \times 10^{-5}$ & $6.6 \times 10^{-8}$ \\
\hline
\end{tabular}

Table 3. Diffusion coefficients of species in water at $25^{\circ} \mathrm{C}$. The values have been corrected for the viscosity with the StokesEinstein equation. ${ }^{4}$

\begin{tabular}{llll}
$D \mathrm{CO}_{2}\left[\mathrm{~m}^{2} \mathrm{~s}^{-1}\right]$ & $D_{\mathrm{HCO}_{3-}}\left[\mathrm{m}^{2} \mathrm{~s}^{-1}\right]$ & $D_{\mathrm{CO}_{32}-}\left[\mathrm{m}^{2} \mathrm{~s}^{-1}\right]$ & $D \mathrm{oH}-\left[\mathrm{m}^{2} \mathrm{~s}^{-1}\right]$ \\
\hline $1.48 \times 10^{-9}$ & $9.25 \times 10^{-10}$ & $7.12 \times 10^{-10}$ & $5.27 \times 10^{-9}$ \\
\hline
\end{tabular}

surface area (ECSA) normalized to the geometric area of the electrode. In the model, ECSA was the surface area of the Ag-electrolyte interface and the geometric area was the hexagonal cross-section of the symmetry unit cell. The film thickness is a function of the number of pores and the pore radius and is shown at end of this section. An electrolyte bulk concentration of $0.1 \mathrm{M} \mathrm{KHCO}_{3}$ was considered with a boundary layer thickness of $1 \mu \mathrm{m}$. This boundary layer thickness has been reported to correspond to a stirring rate of approximately $700-800 \mathrm{rpm}^{14}$ In the concentration boundary layer, the convection and velocity gradients were assumed to be insignificant.

The buffer reactions in the $\mathrm{CO}_{2}$ and bicarbonate system in alkaline solution are:

$$
\begin{array}{ll}
\mathrm{CO}_{2}(\mathrm{aq})+\mathrm{OH}^{-} \Leftrightarrow \mathrm{HCO}_{3}^{-} & K_{1}=4.44 \times 10^{7} 1 / \mathrm{M} \\
\mathrm{HCO}_{3}^{-}+\mathrm{OH}^{-} \Leftrightarrow \mathrm{CO}_{3}^{2-}+\mathrm{H}_{2} \mathrm{O} & K_{2}=4.66 \times 10^{3} 1 / \mathrm{M}
\end{array}
$$

with reaction rate constants from the literature shown in Table $1 .{ }^{20,31}$ The bulk electrolyte is saturated with $\mathrm{CO}_{2}$, and the bulk species concentrations are given in Table $2 .{ }^{14}$ The diffusion coefficients of the various species in water are listed in Table 3. ${ }^{4}$

The following partial differential equations in steady state conditions describe the mass balances of the species in the boundary layer and the pore space:

$$
\begin{aligned}
& 0=D_{\mathrm{CO}_{2}} \nabla^{2}\left[\mathrm{CO}_{2}(\mathrm{aq})\right]-\left[\mathrm{CO}_{2}(\mathrm{aq})\right]\left[\mathrm{OH}^{-}\right] k_{1 \mathrm{f}} \\
& +\left[\mathrm{HCO}_{3}^{-}\right] k_{1 \mathrm{r}} \\
& 0=D_{\mathrm{HCO}_{3}^{-}} \nabla^{2}\left[\mathrm{HCO}_{3}^{-}\right]+\left[\mathrm{CO}_{2}(\mathrm{aq})\right]\left[\mathrm{OH}^{-}\right] k_{1 \mathrm{f}}-\left[\mathrm{HCO}_{3}^{-}\right] k_{1 \mathrm{r}} \\
& -\left[\mathrm{HCO}_{3}^{-}\right]\left[\mathrm{OH}^{-}\right] k_{2 \mathrm{f}}+\left[\mathrm{CO}_{3}^{2-}\right] k_{2 \mathrm{r}} \\
& 0=D_{\mathrm{CO}_{3}^{2-}} \nabla^{2}\left[\mathrm{CO}_{3}^{2-}\right]+\left[\mathrm{HCO}_{3}^{-}\right]\left[\mathrm{OH}^{-}\right] k_{2 \mathrm{f}}-\left[\mathrm{CO}_{3}^{2-}\right] k_{2 \mathrm{r}} \\
& 0=D_{\mathrm{OH}^{-}} \nabla^{2}\left[\mathrm{OH}^{-}\right]-\left[\mathrm{CO}_{2}(\mathrm{aq})\right]\left[\mathrm{OH}^{-}\right] k_{1 \mathrm{f}}+\left[\mathrm{HCO}_{3}^{-}\right] k_{1 \mathrm{r}} \\
& -\left[\mathrm{HCO}_{3}^{-}\right]\left[\mathrm{OH}^{-}\right] k_{2 \mathrm{f}}+\left[\mathrm{CO}_{3}^{2-}\right] k_{2 \mathrm{r}}
\end{aligned}
$$

At the silver electrode surface the two competing reduction reactions are:

$$
\begin{array}{ll}
\mathrm{CO}_{2}+\mathrm{H}_{2} \mathrm{O}+2 e^{-} \Leftrightarrow \mathrm{CO}+2 \mathrm{OH}^{-} & E^{0}=-0.1 \mathrm{~V} \text { vs. RHE (7) } \\
2 \mathrm{H}_{2} \mathrm{O}+2 e^{-} \Leftrightarrow \mathrm{H}_{2}+2 \mathrm{OH}^{-} & E^{0}=0 \mathrm{~V} \text { vs. RHE }
\end{array}
$$

Above, the equilibrium potentials are given versus the reversible hydrogen electrode (RHE). The molar flux of the produced species was calculated from the current densities using Faraday's law. The surface reactions were modeled by concentration dependent Butler-Volmer correlations:

$$
\begin{aligned}
& i_{\mathrm{CO}}=i_{0, \mathrm{CO}}\left(\frac{c_{\mathrm{CO}_{3}^{2-}}}{c_{\mathrm{CO}_{3}^{2}, 0}^{2-0}}\right)^{m}\left(\frac{c_{\mathrm{OH}^{-}}}{c_{\mathrm{OH}^{-}, 0}} \exp \left(\frac{\alpha_{a, \mathrm{CO} F \eta}}{R T}\right)-\frac{c_{\mathrm{CO}_{2}}}{c_{\mathrm{CO}_{2}, 0}} \exp \left(\frac{-\alpha_{c, \mathrm{CO} F} F \eta}{R T}\right)\right) \\
& i_{\mathrm{H}_{2}}=i_{0, \mathrm{H}_{2}}\left(\frac{c_{\mathrm{OH}^{-}, 0}}{c_{\mathrm{OH}^{-}}}\right)^{n}\left(\frac{c_{\mathrm{OH}^{-}}}{c_{\mathrm{OH}^{-}, 0}} \exp \left(\frac{\alpha_{a, \mathrm{H}_{2} F \eta}}{R T}\right)-\exp \left(\frac{-\alpha_{c, \mathrm{H}} F \eta}{R T}\right)\right) \\
& \alpha_{a}+\alpha_{c}=2
\end{aligned}
$$

with exchange current densities, $i_{0}$, anodic, $\alpha_{a}$, and cathodic, $\alpha_{c}$, charge transfer coefficients. With the number of transferred electrons equal to two for both reactions, the charge transfer coefficients in eqs. (9) and (10) can be substituted by $\alpha_{c}=\beta$ and $\alpha_{a}=2-\beta$, and $\beta$ was fitted to experimental data. The anodic back reactions were included in the model, but their contributions were small at the overpotentials relevant for $\mathrm{CO}_{2}$ reduction compared to the cathodic reactions. The mass transport limitations, especially for $\mathrm{CO}_{2}(\mathrm{aq})$, were taken into account with the concentration dependent terms in front of the exponential functions. The water reduction reaction rate has been shown to be intrinsically $\mathrm{pH}$ dependent on $\mathrm{Ag}$ and $\mathrm{Au}$ electrodes. ${ }^{17,19}$ The suppression of the $\mathrm{H}_{2}$ evolution at higher $\mathrm{pH}$ values was modeled with an inverse dependency of the exchange current density on the $\mathrm{OH}^{-}$concentration. The promotion of $\mathrm{CO}$ evolution for mesoporous Ag electrodes has been hypothesized to be related to increased activity of $\mathrm{CO}_{3}{ }^{2-} \cdot{ }^{17,32}$ Our calculations showed (see discussion in section 3.2) that besides the increase in $\mathrm{OH}^{-}$concentration, the activity of $\mathrm{CO}_{3}{ }^{2-}$ was the only activity with a substantial change from the bulk concentration in a mesoporous structure. The parameters $m$ and $n$ of eqs. (9) and (10) were fitted to experimental data. The experimental voltage sweeps of Yoon et al. ${ }^{17}$ showed a limiting current densities for both reduction reactions. For the $\mathrm{H}_{2}$ evolution, the current density reached a plateau at $-0.75 \mathrm{~V} v$ s. RHE for a flat $\mathrm{Ag}$ electrode. In the same study, the CO evolution for a mesoporous Ag electrode with a roughness factor of more than 100 showed a linear increase for higher potentials, indicating a limit in mass transport. Both cases were 
assumed to be caused by adsorption of inhibiting species on the Ag surface and were modeled with an absolute maximum current density:

$$
i=\frac{i_{\mathrm{w}}}{1+\left\|\frac{i_{\mathrm{W}}}{i_{\text {lim }}}\right\|}
$$

where $i_{w}$ is the current density without considering the specific mass transport limitations through adsorption, and $i_{\text {lim }}$ is the limiting current density that correlates to the maximum reaction rate observed in the experiment.

The set of differential eqs. (3)-(6) and algebraic eqs. (7)-(11) was solved on a $3 \mathrm{D}$ tetrahedral mesh using a finite element solver (COMSOL 5.3).

\section{Results}

\subsection{Empirical parameters and model validation}

The exchange current densities, $i_{0}$, and the charge transfer coefficients, $\beta$, for the $\mathrm{CO}_{2}$ and water reduction reactions at the Ag surface were determined by fitting a 3D model to the experimental data of a planar silver electrode in $0.1 \mathrm{M}$ $\mathrm{KHCO}_{3} .{ }^{17}$ The best fitting parameters were found by minimizing the sum of square errors (SSE) between the model and the experimental data for a roughness factor (RF) equal to $4 . \mathrm{RF}=4$ corresponds to a planar electrode and the results are listed in Table 4 and shown in Figure 2. The model was fitted to the averaged experimental results of three planar electrodes. The reduction rate dependencies on the $\mathrm{OH}^{-}$and $\mathrm{CO}_{3}{ }^{-}$activities were initially kept first order. Three Ag-IO with $\mathrm{RF}=43,78,109$ were digitally replicated to

a

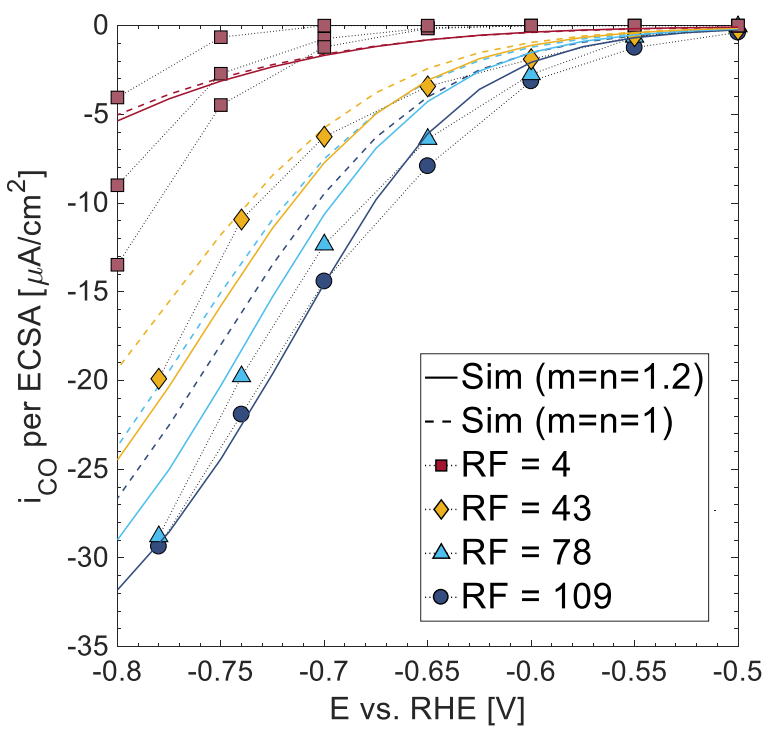

validate the mesoporous model by comparing the simulation results to the experimental values from Yoon et al. ${ }^{17}$ The roughness factor versus film thickness is shown in Figure S1 for the experimental and model structures. With a pore diameter of $200 \mathrm{~nm}$ and channel diameters of $70 \mathrm{~nm}$, the model geometries exhibited smaller RF for the same film thickness. Besides measurement errors, the differences could be caused by a roughness at the pore scale of the Ag10. As the surface area has a dominant effect on the $\mathrm{OH}^{-}$ production and $\mathrm{CO}_{2}$ consumption, the exchange current densities $i_{0, \text { co }}$ and $i_{0, \mathrm{H} 2}$ were multiplied by a correction factor to compensate for the smaller surface area. For both reduction reactions, a limiting current density was observed in the experimental data. The water reduction reached a plateau for a planar electrode at around $-0.75 \mathrm{~V}$ vs. RHE, whereas the $\mathrm{CO}_{2}$ reduction exhibited a linear instead of an exponential increase for RF>43 between -0.7 and $-0.75 \mathrm{~V}$ vs. RHE. The observed mass limiting phenomena could most likely be the adsorption of reaction inhibiting species at the electrolyte-electrode interface, limiting higher current densities. For both reactions, a limiting current density was extracted from the experimental data and the values added to the model are shown in Table 4. The dashed lines in Figure 2 show the simulation results for $m=n=1$. The general trend of $\mathrm{H}_{2}$ evolution suppression and $\mathrm{CO}$ evolution promotion for higher RF can be observed. However, the model is less sensitive to the mesoporous structure; hence, for an $\mathrm{Ag}-\mathrm{IO}$ with $\mathrm{RF}=109$ at a potential of $-0.7 \mathrm{~V}$ vs. RHE, the partial CO current density was 1.6 times smaller and, the

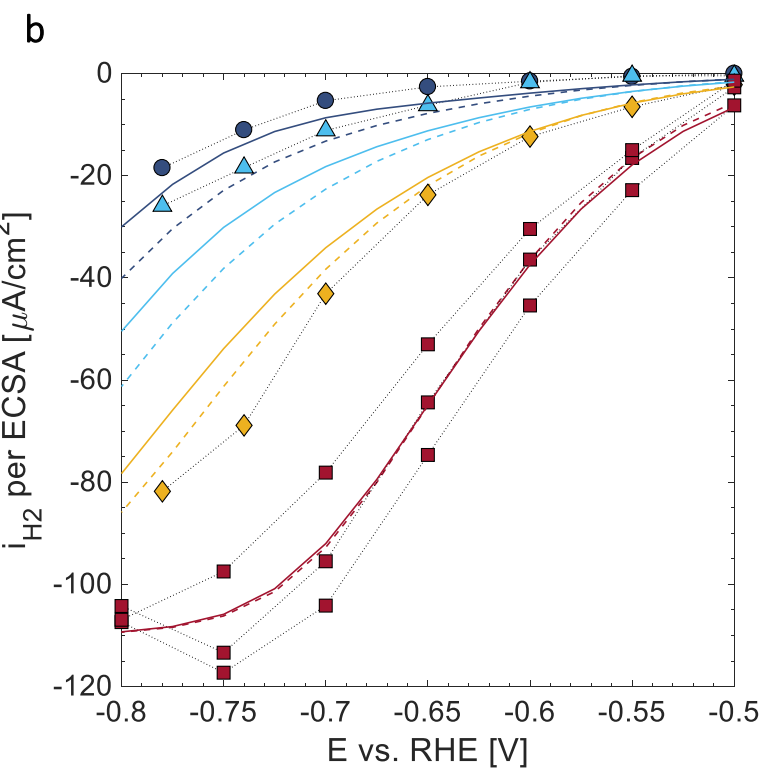

Figure 2. Partial current density for the $\mathrm{CO}_{2}$ and water reduction reaction, normalized by the electrochemical active surface area (ECSA). The experimental data from Yoon et al. ${ }^{17}$ is shown with markers. The computed results are shown with a dashed $(m=n=1)$ and a solid line $(m=n=1.2)$. The lines and markers are color coded based on their roughness factor RF. 


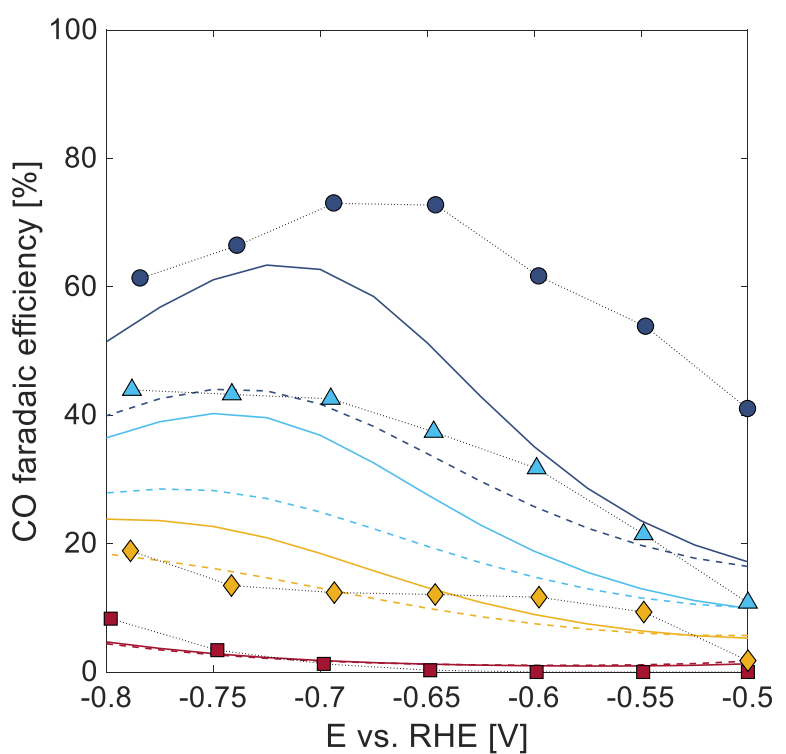

Figure 3. CO faradaic efficiency on Ag films, with differing RF, as a function of applied potential. The legend and color code is the same as in Figure 2.

partial $\mathrm{H}_{2}$ current density was 2.5 times larger than the experimental values. This is also reflected in the faradaic efficiency for the CO production shown in Figure 3, where at the same potential the selectivity towards CO production was 1.7 times smaller than measured experimentally. However, the model predicted the experimentally observed shift to lower potentials for the peak in CO faradaic efficiency, when the roughness of the Ag-IO was increased. In order to increase the sensitivity of the model towards the mesoporous structure, the reduction rate dependencies on the $\mathrm{OH}^{-}$and $\mathrm{CO}_{3}{ }^{-}$activities were allowed to be greater than 1 and fractional. By keeping the same fitting algorithm, the exchange current densities and charge transfer coefficients were determined on the planar 3D simulation for given values of $m$ and $n$. The mesoporous model was then validated and the SSE between the model and the experimental data were assessed. The best fitting values for $m$ and $n$ were found by minimizing the SSE. The parameters are listed in Table 4 and, the partial current densities are shown with a solid line in Figure 2. For an Ag-IO with RF=109 at a potential of $-0.7 \mathrm{~V}$ vs. RHE, the model partial CO current density matched the experimental value, and the partial $\mathrm{H}_{2}$ current density was 1.6 times larger than measured. The model, with $m=1.2$ and $n=1.2$, predicted a maximum faradaic efficiency in the CO production of $63 \%$ at $-0.725 \mathrm{~V}$ vs. RHE, 10 percentage points less than measured with a shift in potential of $-0.025 \mathrm{~V}$. The goodness of fit was quantified by the R-square method and the values for both parameter sets are shown in Table 4. The model with the parameter set 2 described $81 \%$ and $79 \%$ of the total variation in the experimental data for the $\mathrm{CO}$ and $\mathrm{H}_{2}$ evolution, respectively. The parameter set 2 was used in the mesoporous model for the subsequent sections.

A sensitivity study was done by independently varying the pore and channel diameter by $\pm 10 \%$ (Figure S2). Both parameters altered the suppression of the water reduction reaction. Reducing the pore diameter decreased the CO selectivity, whereas a reduction in the channel diameter enhanced the CO selectivity. In this sensitivity study, the number of pores were kept constant, but the film thickness changed, leading to a smaller overall surface area for a reduced pore diameter, and a larger surface area for a structure with smaller channel diameters. For both cases, the deviation in the maximum CO faradaic efficiencies was smaller than 7 percent points.

\subsection{Local current densities and species concentrations}

The validated 3D model of the replicated mesoporous Ag-IO offers unique insights into the local current density distributions of the $\mathrm{CO}_{2}$ and water reduction reactions and the local concentrations of the species $\mathrm{CO}_{2}, \mathrm{OH}^{-}, \mathrm{HCO}_{3}{ }^{-}$, and $\mathrm{CO}_{3}{ }^{2-}$ in the pore space. Overall, the partial $\mathrm{CO}$ current per ECSA was promoted for Ag-IO with higher roughness factors, and the partial $\mathrm{H}_{2}$ current was suppressed. However, the local current densities were not homogenously distributed along the z-axis, as shown in Figure 4 a for a potential of $-0.8 \mathrm{~V}$ vs. RHE.

The biggest suppression of $\mathrm{H}_{2}$ evolution occurred in the lowest $20 \%$ of the pore network, whereas in the shallower pores, near $z=t$, the $\mathrm{H}_{2}$ partial current density increased sharply to values close to the planar case. The promotion for the $\mathrm{CO}$ evolution was also more pronounced near the bottom of the electrode, with a small decrease in CO partial current density towards the top of the film. These observations were consistent with the calculated species concentrations shown in Figure $4 \mathrm{~b}$. The rate of $\mathrm{OH}^{-}$ production in the deepest pores was substantially higher than the diffusion flux towards the bulk electrolyte. This led

Table 4. Empirical parameters fitted to the experimental data of a planar Ag electrode. $R^{2}$ quantified the goodness of fit for both sets.

\begin{tabular}{cccccccccccc} 
Set & $i_{0, \mathrm{CO}}\left[\mathrm{A} \mathrm{m}{ }^{-2}\right]$ & $i_{0, \mathrm{H}_{2}}\left[\mathrm{~A} \mathrm{~m}^{-2}\right]$ & $i_{\text {lim, } \mathrm{CO}}\left[\mathrm{A} \mathrm{m}{ }^{-2}\right]$ & $i_{\text {lim, } \mathrm{H}_{2}}\left[\mathrm{~A} \mathrm{~m}^{-2}\right]$ & $\beta_{\mathrm{CO}}[-]$ & $\beta_{\mathrm{H}_{2}}[-]$ & $m[-]$ & $n[-]$ & $R^{2}{ }_{\mathrm{CO}}[\%]$ & $R^{2}{ }_{\mathrm{H}_{2}}[\%]$ \\
\hline 1 & $1.0012 \times 10^{-5}$ & $7.0102 \times 10^{-10}$ & 0.4 & 1.1 & 0.29 & 0.95 & 1 & 1 & 71 & 68 \\
2 & $8.4083 \times 10^{-6}$ & $9.2084 \times 10^{-10}$ & 0.4 & 1.1 & 0.29 & 0.95 & 1.2 & 1.2 & 81 & 79 \\
\hline
\end{tabular}


a

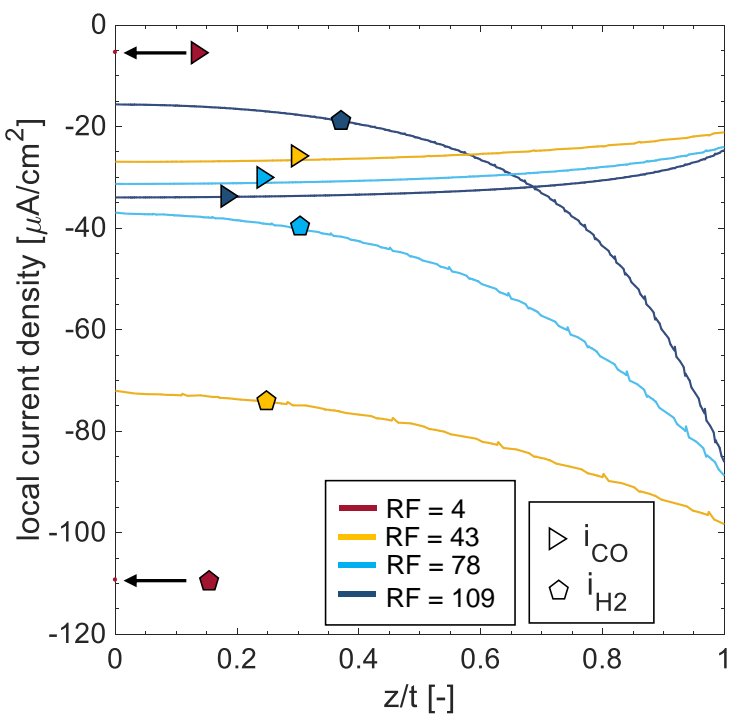

b

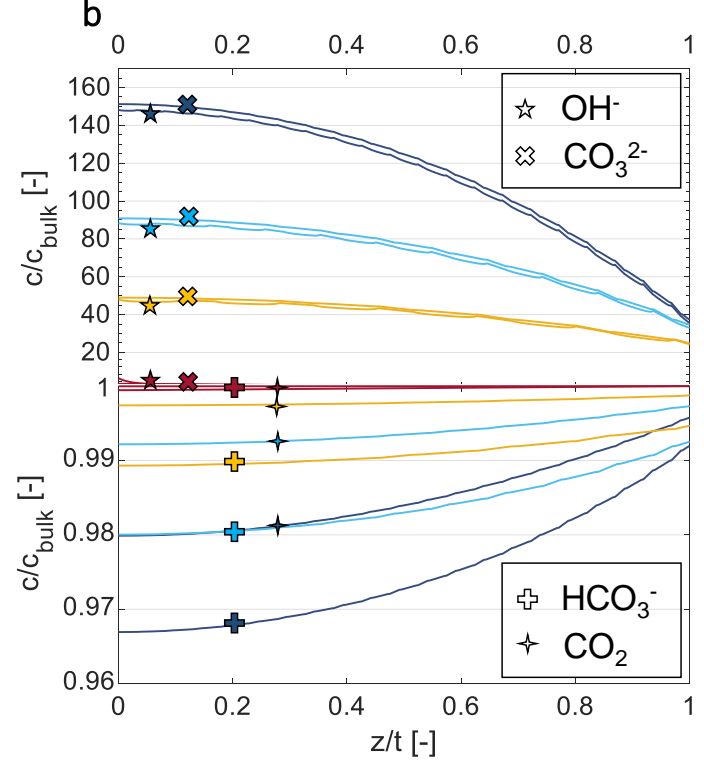

Figure 4. (a) Local current density profiles of $\mathrm{CO}$ and $\mathrm{H}_{2}$ evolution along the $z$-axis of $\mathrm{Ag}$-IO electrodes with $\mathrm{RF}=4,43,78,109$ and thickness $t=0$ (planar), 1.7, 3.1, $6.2 \mu \mathrm{m}$, respectively. (b) Concentration profiles along the $z$-axis of $\mathrm{OH}^{-}, \mathrm{CO}_{3}{ }^{2}, \mathrm{HCO}_{3}{ }^{-}$and $\mathrm{CO}_{2}$ versus their bulk concentration. Both Figures are at a potential of $-0.8 \mathrm{~V}$ vs. RHE.

to a build-up of $\mathrm{OH}^{-}$and, through the buffer reaction of the electrolyte, also increased the $\mathrm{CO}_{3}{ }^{2-}$ concentration. For pores closer to the top of the film, the mass transport towards the bulk electrolyte was faster, leading to lower $\mathrm{OH}^{-}$ and $\mathrm{CO}_{3}{ }^{2-}$ concentrations. The calculations showed that the top $40 \%$ of the Ag-IO films substantially reduced the selectivity towards the $\mathrm{CO}$ evolution and that higher $\mathrm{CO}$ faradaic efficiencies could be achieved by decreasing the mass transfer in this part of the film.

Figure $\mathrm{S} 3$ proves that there are no concentration gradients perpendicular to the z-axis for $\mathrm{CO}_{2}, \mathrm{HCO}_{3}{ }^{-}$, and $\mathrm{CO}_{3}{ }^{2}$ and only a small gradient for $\mathrm{OH}( \pm 0.3 \%)^{-}$. Also, the $\mathrm{OH}^{-}$concentration was the only concentration profile that was not monotonically increasing or decreasing along the z-axis in the pore space. At the top section of each pore, the $\mathrm{OH}^{-}$ concentration increased slightly due to the longer diffusion path through one of the three channels that are not on the top of the pore space. This explains the wavy $\mathrm{OH}^{-}$ concentration profile in Figure 4b.

For the considered operation conditions, the consumption of $\mathrm{CO}_{2}$ was low and with a maximum decrease of $2 \%$ from the saturation concentration ( $R F=109)$, far away from mass transport limitations. Also the depletion of $\mathrm{HCO}_{3}{ }^{-}$was small and has been shown not to take part in any rate-limiting step $^{14}$.

\subsection{Pore size vs. film thickness}

The film thickness, $t$, and the pore size, $d_{\text {pore }}$, determine the morphology of inverse opal mesoporous structures. When using a self-assembling sphere packing for the template, the pore network is defined by an FCC lattice in a hexagonal close-packed arrangement. ${ }^{33}$ A roughness factor of 1 was assumed on the pore level and the dependency of RF vs. $t$ was linear (Figure S1). The sphere overlap, $d r$, from the sintering process, was assumed to be proportional to the pore radius. The relation $d r=0.07 \cdot r_{\text {pore }}$ was extracted from SEM images of the Ag-IO. ${ }^{17}$ Therefore, the surface area, the roughness factor and, the tortuosity are geometrically linked to $t$ and $d_{\text {pore }}$.

Increasing the film thickness for a constant pore size increased the diffusion path lengths of the species and more $\mathrm{OH}^{-}$and $\mathrm{CO}_{3}{ }^{2-}$ accumulated in the pore network. As previously seen, this led to a higher partial CO current densities and a higher CO faradaic selectivity. Figure 5a shows the partial CO current density, and Figure 5b shows the CO faradaic selectivity for Ag-IO of thickness 3, 6 and 15 $\mu \mathrm{m}$. In contrary, increasing the pore size for a constant film thickness decreased both current density and selectivity. Having bigger pores for the same film thickness translates to a smaller surface area, and therefore, a lower rate of $\mathrm{OH}^{-}$ production which favors the water reduction reaction. For the considered pore diameters of $100-400 \mathrm{~nm}$, the change in the averaged tortuosity was negligible as shown in Figure 6 . With the thickness of the film much bigger than the pore diameter, mass transport lateral to the film thickness was insignificant.

Figure $5 c$ and $5 d$ quantify the effect of the film thickness and the pore diameter on the maximal achievable CO faradaic efficiency and the required electrode potential. The linear dependency of RF vs. $t$ and the non-linear dependency of RF 
a

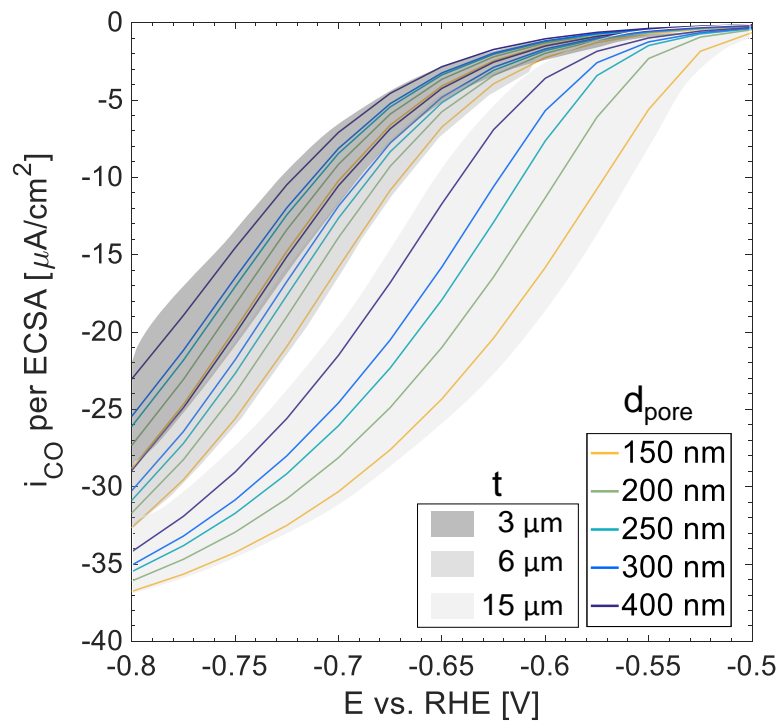

C

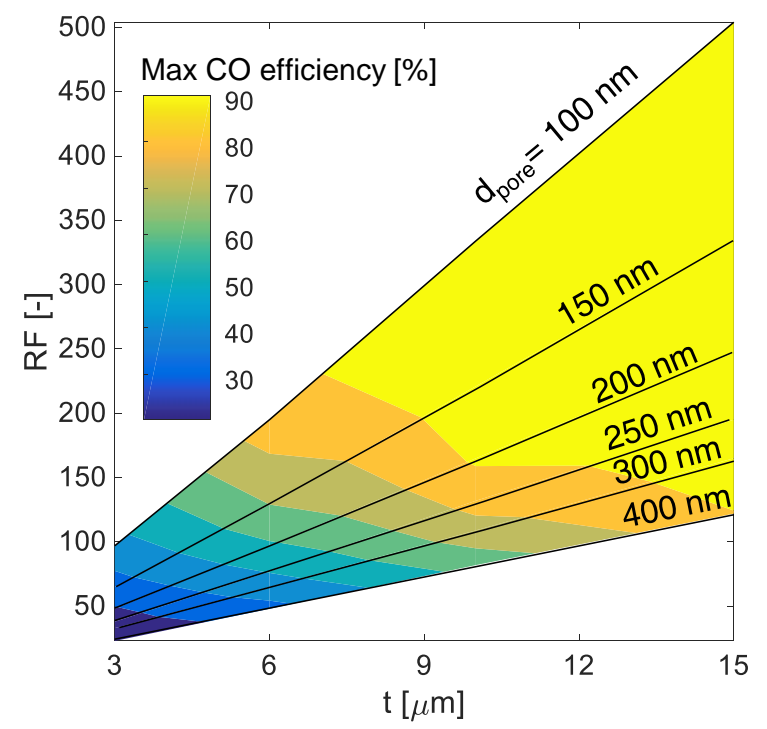

b

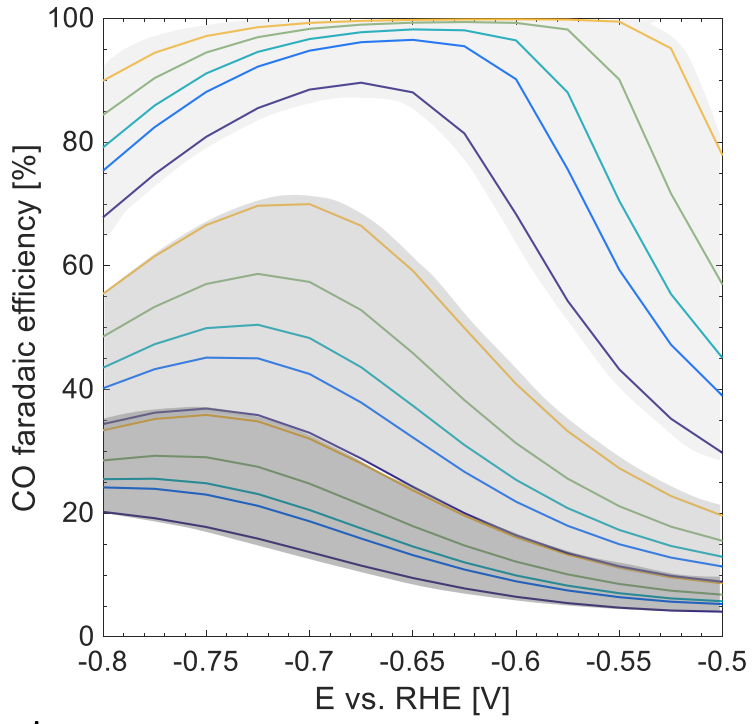

d

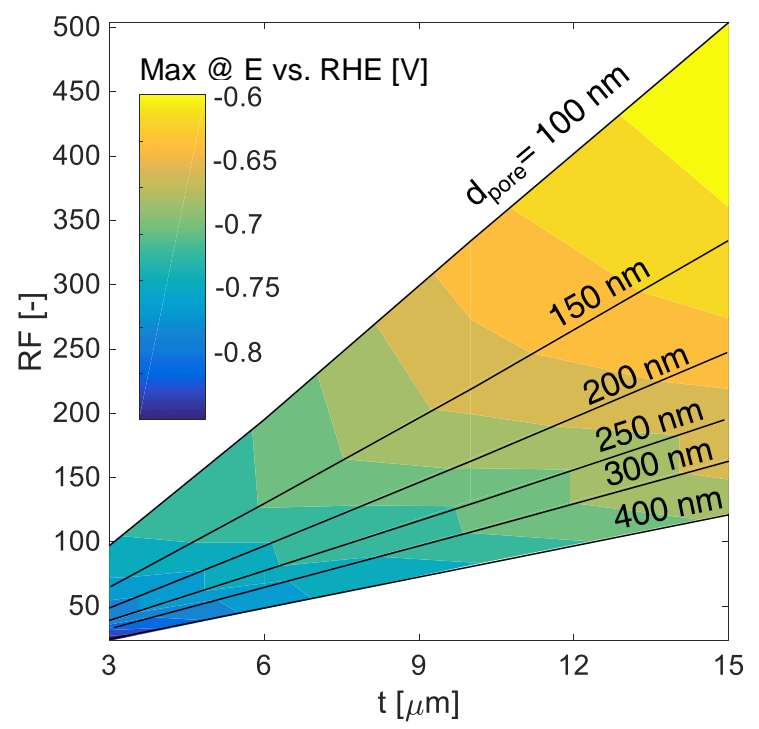

Figure 5. (a) Partial CO current density and (b) CO faradaic efficiency for Ag-IO with pore diameters of 150-400 nm and film thickness of 3, 6 and $15 \mu \mathrm{m}$. (c) The values of the maximum CO faradaic efficiency in the contour plot as a function of $t$ and RF. The linear relation between RF and $t$ is shown for the different pore diameters. (d) The potentials vs. RHE for the maximum CO faradaic efficiency as a function of the film thickness $t$ and the roughness factor RF.

vs. $d_{\text {pore }}$ is shown. For a pore diameter of $200 \mathrm{~nm}$, a film thickness of more than $10 \mu \mathrm{m}$ was needed to push the CO faradaic efficiency above $90 \%$. The same faradaic efficiency could be achieved with a pore diameter of $100 \mathrm{~nm}$ at a reduced film thickness of $7 \mu \mathrm{m}$. The thin film consisted of 93 pores, whereas the thicker film only had 67 pores. With the porosity independent of the pore size at a value of $0.87,30 \%$ of Ag could be saved by halving the pore size. From this point of view, reducing the pore size is the superior path to boost the selectivity towards $\mathrm{CO}_{2}$ reduction. However, in reality, the optimum for such an electrode will also depend on the homogeneity of the mesoporous film. Whether thin films with small pore diameters or thicker films with big pore diameters can be fabricated with a higher homogeneity and reproducibility needs to be experimentally investigated. In summary, for 10 films, it is not enough to characterize the film by its roughness factor; instead, the film thickness and pore diameter were observed to be the key parameters to optimize the geometry.

In general, the peak of the maximum CO faradaic efficiency was observed at lower potentials vs. RHE when increasing the film thickness or reducing the pore diameter. The potential was linked to the value of the maximum CO faradaic efficiency, and not directly dependent on the film thickness and pore diameter. Both film mentioned above $\left(t=10 \mu \mathrm{m}, d_{\text {pore }}=200 \mathrm{~nm}\right.$ and $t=7 \mu \mathrm{m}, d_{\text {pore }}=100 \mathrm{~nm}$ ) have a maximum CO faradaic efficiency of $90 \%$ at the same potential of $-0.675 \mathrm{~V}$ vs. RHE. Using a mesoporous Ag-IO not 


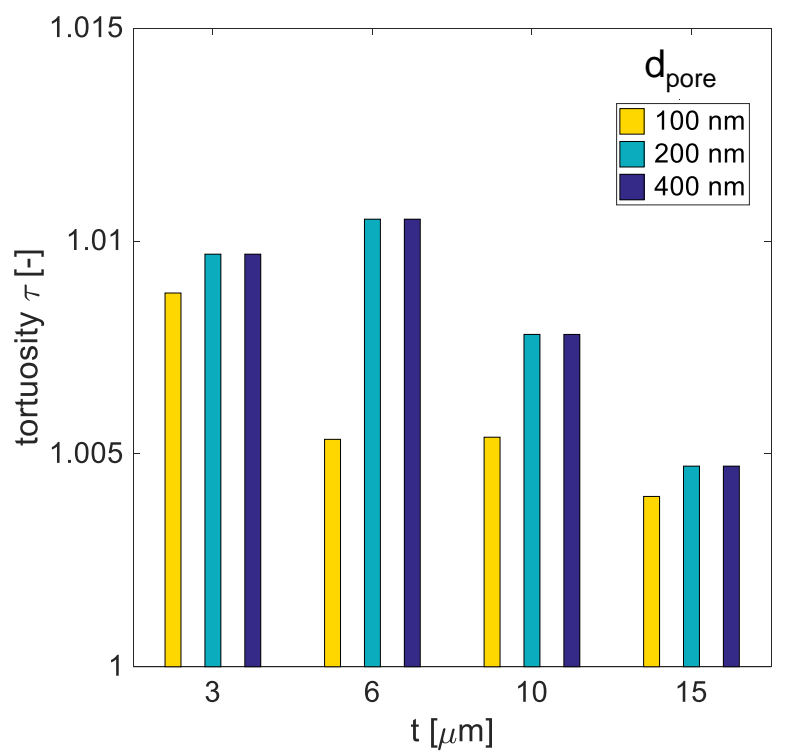

Figure 6 . The averaged tortuosity as a function of film thickness $(3,6,10$, and $15 \mu \mathrm{m})$ and pore diameter $(100,200$ and $400 \mathrm{~nm})$. The tortuosity is defined as the diffusion path length through the entire Ag-IO film divided by the thickness $t$.

only enhances substantially the CO faradaic efficiency, but also decreases the required potentials vs. RHE.

\subsection{Additional diffusion layer}

As shown in section 3.2, a substantial part of the selectivity towards the $\mathrm{CO}_{2}$ reduction was lost in the top half of the Ag-

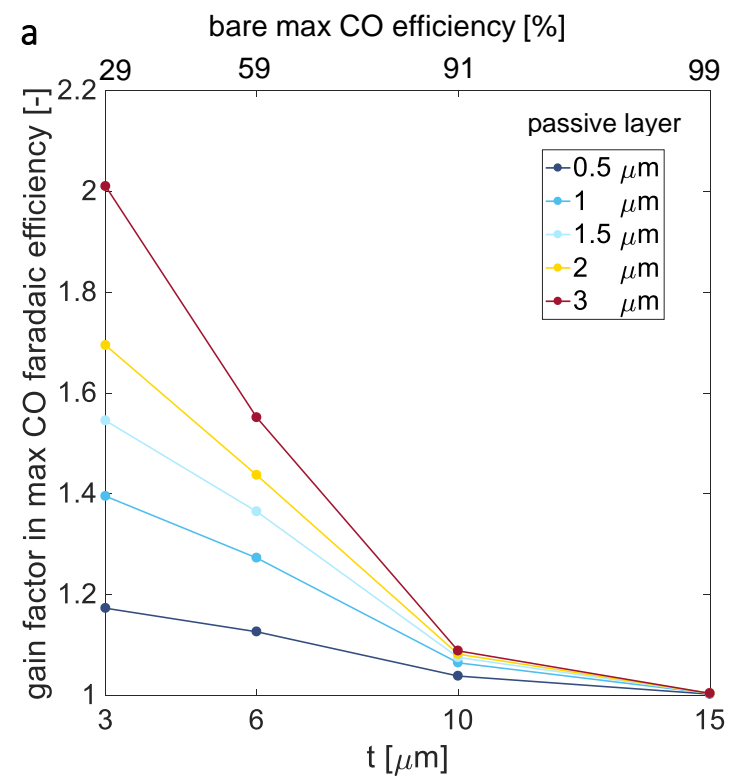

$\mathrm{IO}$, where local concentrations of $\mathrm{OH}^{-}$and $\mathrm{CO}_{3}{ }^{2-}$ were not high enough to suppress the $\mathrm{H}_{2}$ or promote the $\mathrm{CO}$ production, respectively. With thicker films or smaller pore diameters, the contributions of the top part of the mesoporous film relative to the overall fuel production of the film were smaller, but the aforementioned phenomena remained. In order to address this issue, an additional chemically inert diffusion layer was introduced on top of the $\mathrm{Ag}-\mathrm{IO}$. From an experimental point of view, the most straightforward approach would be an inert layer using the same polystyrene sphere template as for the Ag film. After the electrochemical deposition of $\mathrm{Ag}$, another metal layer, with no activity towards $\mathrm{CO}_{2}$ and water reduction, could be deposited in the same manner. For the calculations, pore diameters of $200 \mathrm{~nm}$ were considered and a roughness factor of 1 was assumed on the pore level. Zero current densities at the interface of the electrolyte and the inert layer was assumed Figure 7a shows the gain factor in the maximum $\mathrm{CO}$ faradaic efficiency for Ag films with a thickness of 3, 6, 10 and $15 \mu \mathrm{m}$, and a layer thickness of the inert metal of $0.5,1,1.5,2$ or $3 \mu \mathrm{m}$. Evidently, the thick Ag films with the additional diffusion layer, and with an already high CO faradaic efficiency, had less of a gain compared to the thinner Ag films. For the latter, a dramatic improvement was observed. For a $3 \mu \mathrm{m}$ thick Ag-IO, the CO faradaic efficiency could be increased from $29 \%$ to over $58 \%$ by adding $3 \mu \mathrm{m}$ of an inert layer. Whereas with a $6 \mu \mathrm{m}$ thick Ag film, the improvement was less drastic, yet the efficiency could be pushed above $91 \%$. Figure $7 \mathrm{~b}$ shows the effect of the

b

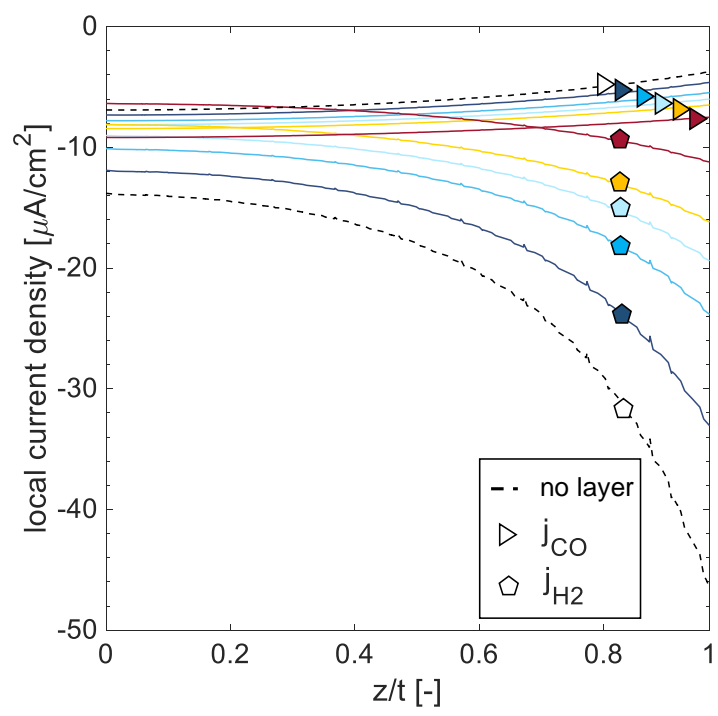

Figure 7. (a) The gain factor in the maximum CO faradaic efficiency resulting from an additional chemically inert layer with the same mesoporous structure on top of the Ag-IO. The added layer has a thickness between 0.5 and $3 \mu$ m. The maximum CO faradaic efficiency of the bare electrode (without an inert layer) is shown on the top x-axis. (b) The local $\mathrm{CO}$ and $\mathrm{H}_{2}$ evolution current density distribution along the $z$-axis for a $t=3 \mu \mathrm{m}$ thick $\mathrm{Ag}-\mathrm{IO}$ at $-0.7 \mathrm{~V}$ vs. RHE with (colored lines) and without (dashed line) the chemically inert layer of thickness between 0.5 and $3 \mu \mathrm{m}$. Local current densities at the film position $z$ were averaged. 
thickness of the inert top layer on the local current density distributions for a $3 \mu \mathrm{m}$ thick $\mathrm{Ag}-\mathrm{IO}$ at $-0.7 \mathrm{~V}$ vs. RHE. Local current densities at the film position $z$ were averaged. The 3 $\mu m$ thick diffusion layer limited the increase in the $\mathrm{H}_{2}$ partial current density from the bottom of the electrode to the top to $75 \%$, whereas it increased by $236 \%$ for the bare Ag film. The introduction of an additional diffusion layer successfully suppresses the water reduction in the shallower pores of the Ag film. In addition, the CO faradaic efficiency is further improved throughout the whole electrode due to the increased diffusion path lengths.

The concept of an additional diffusion layer on top of the AgIO can be extended by considering lower porosities, higher tortuosity or thicker films of the inert material. A porous

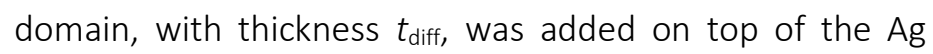
structure in our model. Within that porous domain, the diffusion coefficients were reduced by a factor $p$, resulting in the effective diffusion coefficient, $D_{\text {eff: }}$ :

$$
D_{\text {eff }}=p \cdot D \quad(\text { e.g. } \quad p=\varepsilon / \tau<1)
$$

The reduction of the effective diffusion coefficient, if not experimentally measured, can be estimated from the ratio of the porosity and tortuosity. ${ }^{34}$ The porosity accounts for the reduced electrolyte volume fraction and the tortuosity for the longer diffusion pathlengths through the porous domain. As seen in Figure 6, the tortuosities of inverse-opal layers are close to one, leading to $p \approx \varepsilon$. Inside the porous

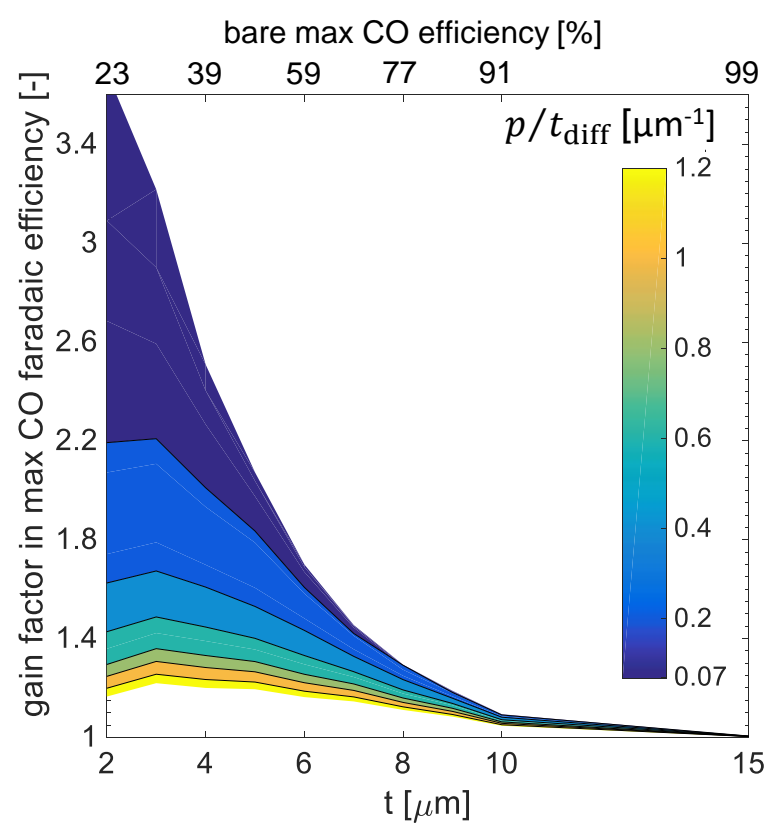

Figure 8. The gain factor in the maximum CO faradaic efficiency resulting from an additional porous inert diffusion layer on top of the Ag-IO. $p(<1)$ is the factor by which the diffusion coefficients are reduced in the porous media and $t_{\text {diff }}$ is the thickness of the diffusion layer. material of thickness $t_{\text {diff, }}$ the buffer reactions were still considered. The mass transport through the porous layer was reduced by either decreasing $p$ or increasing the thickness $t_{\text {diff. }}$ Figure 8 quantifies the gain factor in the maximum CO faradaic efficiency as a function of the ratio of those two parameters. As a comparison, the inert metal layers (Figure 7a) with a thickness of $0.5 \mu \mathrm{m}$ or $3 \mu \mathrm{m}$, correspond to a ratio $p / t_{\text {diff }}$ of 1.74 or 0.29 , respectively. Ratios larger than 1.2 are not shown in the contour plot, as their gain factors were small and not of interest. Figure 8 can be used as a design guideline to choose an appropriate diffusion layer on top of a mesoporous $\mathrm{Ag}-1 \mathrm{O}$ to boost the CO faradaic efficiency above $90 \%$ for a given film thickness. For an optimum $\mathrm{CO}_{2}$ reduction electrode, the film thickness should be as large as experimentally possible to benefit from the large surface area, combined with the right diffusion layer to suppress the water splitting reaction in the upper part of the catalytic film.

\section{Conclusion}

We developed a finite element numerical model applied to an accurate 3D geometrical representation of an ordered mesostructured Ag electrode. The model takes into account the mass transport and the electrolyte buffer reactions in the pore space and predicts the catalytic reaction rates at the electrolyte-Ag interface based on concentration dependent Butler-Volmer correlations. We demonstrated that the dramatic suppression of the undesirable $\mathrm{H}_{2}$ evolution reaction rate and the promotion of the $\mathrm{CO}$ evolution reaction rate as a function of the roughness factor could successfully be modeled by an inverse $\mathrm{OH}^{-}$ concentration dependency of the $\mathrm{H}_{2}$ evolution exchange currency and a proportional dependency of the $\mathrm{CO}$ evolution exchange currency on the $\mathrm{CO}_{3}{ }^{2-}$ concentration. The best agreement with experimental data was found for fractional reaction orders of 1.2 for both evolution rates. The model gives detailed insights on the local concentration distributions of $\mathrm{CO}_{2}(\mathrm{aq}), \mathrm{OH}^{-}, \mathrm{HCO}_{3}{ }^{-}$, and $\mathrm{CO}_{3}{ }^{2-}$ in the pore space. For a $0.1 \mathrm{M} \mathrm{KHCO}_{3}$ electrolyte, saturated with $\mathrm{CO}_{2}$, the considered operation conditions for a maximum $\mathrm{CO}$ faradaic selectivity were far away from a mass transport limit of $\mathrm{CO}_{2}$ (aq). However, a substantial build-up of $\mathrm{OH}^{-}$and $\mathrm{CO}_{3}{ }^{2}$ was observed in the lower part of the film, as the mass transport rate could not keep up with the $\mathrm{OH}^{-}$evolution rate. In contrast, the shallower pores exhibited low $\mathrm{OH}^{-}$and $\mathrm{CO}_{3}{ }^{2}$ concentrations that were translated in locally high $\mathrm{H}_{2}$ and low CO current densities. Therefore, a significant part of the potential for a high CO faradaic efficiency of a mesostructured Ag electrode was lost in the top half of the 
catalytic film, due to a high mass transport rate. The morphology of the electrode, which the species need to diffuse through, could be described solely by the pore diameter. The pores were always stacked in a hexagonal close-packed arrangement in an FCC lattice, which was given by the colloidal polystyrene spheres template. The maximal achievable $\mathrm{CO}$ selectivity was dictated by the pore diameter and the film thickness. Both parameters directly influenced the surface area, which was the key property to boost the $\mathrm{CO}$ faradaic efficiency. The goal is to have as much active $\mathrm{Ag}$ surface in regions where the $\mathrm{OH}^{-}$and $\mathrm{CO}_{3}{ }^{2}$ concentrations are high. For a mesostructured $\mathrm{Ag}$ electrode with a pore diameter of $200 \mathrm{~nm}$, a thickness of at least 10 $\mu \mathrm{m}$ is required to reach a CO selectivity above $90 \%$. The film thickness can be substantially reduced when using a template with smaller spheres, as the surface area per film thickness is increased. A reduced film thickness could lead to more homogeneous deposition of $\mathrm{Ag}$ during the fabrication process of the electrode.

Based on the detailed insights from the model, we proposed a strategy to further increase the CO faradaic efficiency of a mesostructured $\mathrm{Ag}$ electrode. To address the low $\mathrm{H}_{2}$ evolution rate suppression in shallow pores and to reduce the amount of expensive catalytic material, an additional chemically inert diffusion layer can be introduced on top of the $\mathrm{Ag}$ electrode. Using the morphology from the same template, but depositing an inert material on top, the CO selectivity was successfully increased from $60 \%$ to more than $90 \%$. We generalized the approach by characterizing the diffusion layer as a porous inert media with a certain thickness and reduced effective diffusion coefficients. The effect of the layer thickness and the reduction factor of the diffusion coefficient on the $\mathrm{CO}$ selectivity was predicted by our model.

The results of our mass transport model provide rational design guidelines for the fabrication of ordered, mesostructured $\mathrm{Ag}$ electrodes for the selective, and efficient $\mathrm{CO}_{2}$ reduction reaction. In the considered parameter range, the electrode should be as thick as experimentally possible to ensure a high total CO evolution current, while a reduced pore size and the addition of an inert diffusion layer can dramatically boost the CO selectivity.

The model can be applied to other material, e.g. $\mathrm{Cu}$, in order to investigate and provide guidelines for more selective mesoporous layers. However, for each material the empirical parameters in the Butler-Volmer correlations need to be quantified beforehand in order to apply the model. Eventually, multi-layered mesostructured electrodes could be proposed, designed and optimized for the selective processing of other $\mathrm{CO}_{2}$ reduction products by, for example, introducing a multi-step process in which the selective $\mathrm{CO}$ evolution on the Ag surface could be followed by selective conversion to hydrocarbons on a Cu surface.

\section{Acknowledgments}

We thank Youngmin Yoon and Yogesh Surendranath for the helpful discussions. This material is based upon work performed with the financial support of the starting grant of the Swiss National Science Foundation, as part of the SCOUTS project (grant \#155876), and of the Hans-Eggenberger Foundation. 
1. Tang, W. et al. The importance of surface morphology in controlling the selectivity of polycrystalline copper for CO2 electroreduction. Phys. Chem. Chem. Phys. 14, 76-81 (2012).

2. Kuhl, K. P. et al. Electrocatalytic conversion of carbon dioxide to methane and methanol on transition metal surfaces. J. Am. Chem. Soc. 136, 14107-14113 (2014).

3. Jones, J.-P., Prakash, G. K. S. \& Olah, G. A. Electrochemical CO2 Reduction: Recent Advances and Current Trends. Isr. J. Chem. 54, 1451-1466 (2014).

4. Hori, Y. in (eds. Vayenas, C. G., White, R. E. \& Gamboa-Aldeco, M. E.) 89-189 (Springer, 2008). doi:10.1007/978-0-387-49489-0_3

5. Olah, G. A., Prakash, G. K. S. \& Goeppert, A. Anthropogenic chemical carbon cycle for a sustainable future. J. Am. Chem. Soc. 133, 12881-12898 (2011).

6. Whipple, D. T. \& Kenis, P. J. A. Prospects of $\mathrm{CO} 2$ utilization via direct heterogeneous electrochemical reduction. J. Phys. Chem. Lett. 1, 3451-3458 (2010).

7. Singh, M. R., Clark, E. L. \& Bell, A. T. Thermodynamic and achievable efficiencies for solar-driven electrochemical reduction of carbon dioxide to transportation fuels. Proc. Natl. Acad. Sci. 112, E6111-E6118 (2015).

8. Durst, J. et al. Electrochemical CO2 Reduction - A Critical View on Fundamentals, Materials and Applications. Chim. Int. J. Chem. 69, 769-776 (2015).

9. Gattrell, M., Gupta, N. \& Co, A. A review of the aqueous electrochemical reduction of CO2 to hydrocarbons at copper. J. Electroanal. Chem. 594, 1-19 (2006).

10. Gutierrez, R. R. \& Haussener, S. Modeling of Concurrent CO2 and Water Splitting by Practical Photoelectrochemical Devices. J. Electrochem. Soc. 163, H1008-H1018 (2016).

11. Peterson, A. A. \& Nørskov, J. K. Activity descriptors for $\mathrm{CO} 2$ electroreduction to methane on transition-metal catalysts. J. Phys. Chem. Lett. 3, 251-258 (2012).

12. Kuhl, K. P., Cave, E. R., Abram, D. N. \& Jaramillo, T. F. New insights into the electrochemical reduction of carbon dioxide on metallic copper surfaces. Energy Environ. Sci. 5, 7050-7059 (2012).

13. Li, C. W. \& Kanan, M. W. CO2 reduction at low overpotential on Cu electrodes resulting from the reduction of thick Cu2O films. J. Am. Chem. Soc. 134, 7231-7234 (2012).

14. Wuttig, A., Yoon, Y., Ryu, J. \& Surendranath, Y. Bicarbonate Is Not a General Acid in Au-Catalyzed CO2 Electroreduction. J. Am. Chem. Soc. 139, 17109-17113 (2017).

15. Hori, Y., Ito, H., Okano, K., Nagasu, K. \& Sato, S. Silver-coated ion exchange membrane electrode applied to electrochemical reduction of carbon dioxide. Electrochim. Acta 48, 2651-2657 (2003).

16. Lu, Q. et al. A selective and efficient electrocatalyst for carbon dioxide reduction. Nat. Commun. 5, 1-6 (2014).

17. Yoon, Y., Hall, A. S. \& Surendranath, Y. Tuning of Silver Catalyst Mesostructure Promotes Selective Carbon Dioxide Conversion into Fuels. Angew. Chemie - Int. Ed. 55, 15282-15286 (2016).

18. Mistry, H., Varela, A. S., Kühl, S., Strasser, P. \& Cuenya, B. R. Nanostructured electrocatalysts with tunable activity and selectivity. Nat. Rev. Mater. 1, (2016). 
19. Hall, A. S., Yoon, Y., Wuttig, A. \& Surendranath, Y. Mesostructure-Induced Selectivity in CO2 Reduction Catalysis. J. Am. Chem. Soc. 137, 14834-14837 (2015).

20. Gupta, N., Gattrell, M. \& MacDougall, B. Calculation for the cathode surface concentrations in the electrochemical reduction of $\mathrm{CO} 2$ in KHCO3 solutions. J. Appl. Electrochem. 36, 161-172 (2006).

21. Suter, S., Cantoni, M., Gaudy, Y. K., Pokrant, S. \& Haussener, S. Linking morphology and multiphysical transport in structured photoelectrodes. Sustain. Energy Fuels 2661-2673 (2018). doi:10.1039/C8SE00215K

22. Hori, Y., Murata, A. \& Takahashi, R. Formation of hydrocarbons in the electrochemical reduction of carbon dioxide at a copper electrode in aqueous solution. J. Chem. Soc., Faraday Trans. 1 85, 2309-2326 (1989).

23. Ooka, H., Figueiredo, M. C. \& Koper, M. T. M. Competition between Hydrogen Evolution and Carbon Dioxide Reduction on Copper Electrodes in Mildly Acidic Media. Langmuir 33, 9307-9313 (2017).

24. Schouten, K. J. P., Pérez Gallent, E. \& Koper, M. T. M. The influence of pH on the reduction of CO and CO2 to hydrocarbons on copper electrodes. J. Electroanal. Chem. 716, 53-57 (2014).

25. Singh, M. R., Kwon, Y., Lum, Y., Ager, J. W. \& Bell, A. T. Hydrolysis of Electrolyte Cations Enhances the Electrochemical Reduction of CO2 over Ag and Cu. J. Am. Chem. Soc. 138, 13006-13012 (2016).

26. Singh, M. R., Clark, E. L. \& Bell, A. T. Effects of electrolyte, catalyst, and membrane composition and operating conditions on the performance of solar-driven electrochemical reduction of carbon dioxide. Phys. Chem. Chem. Phys. 17, 18924-18936 (2015).

27. Weng, L. C., Bell, A. T. \& Weber, A. Z. Modeling gas-diffusion electrodes for CO2 reduction. Phys. Chem. Chem. Phys. 20, 16973-16984 (2018).

28. Burdyny, T. \& Smith, W. A. CO2 reduction on gas-diffusion electrodes and why catalytic performance must be assessed at commercially-relevant conditions. Energy Environ. Sci. (2019). doi:10.1039/C8EE03134G

29. Hashiba, H. et al. Effects of electrolyte buffer capacity on surface reactant species and the reaction rate of CO2 in Electrochemical CO2 reduction. J. Phys. Chem. C122, 3719-3726 (2018).

30. Raciti, D., Mao, M. \& Wang, C. Mass transport modelling for the electroreduction of CO2 on Cu nanowires. Nanotechnology 29, 044001 (2018).

31. Sullivan, B. P., Krist, K. \& Guard, H. E. Electrochemical and electrocatalytic reactions of carbon dioxide. (Elsevier, 2012).

32. Verma, S., Lu, X., Ma, S., Masel, R. I. \& Kenis, P. J. A. The effect of electrolyte composition on the electroreduction of CO2 to CO on Ag based gas diffusion electrodes. 7075-7084 (2016). doi:10.1039/c5cp05665a

33. Hung, D., Liu, Z., Shah, N., Hao, Y. \& Searson, P. C. Finite Size Effects in Ordered Macroporous Electrodes Fabricated by Electrodeposition into Colloidal Crystal Templates. J. Phys. Chem. C111, 3308-3313 (2007).

34. Cussler, E. L. in Diffusion: Fundamentals of Mass Transfer 237-273 (Cambridge University Press, 2011). doi:10.1017/CBO9780511805134.010 

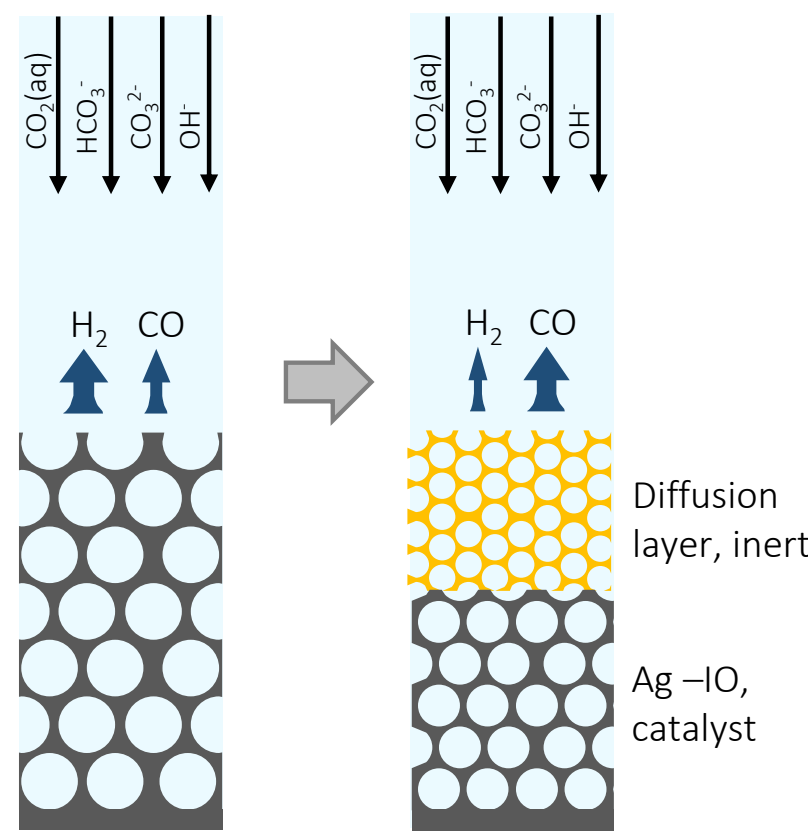

For Table of Contents Only 